Please do not remove this page

RMIT

UNIVERSITY

\title{
Measurement of glucose exclusion from the fully hydrated DOPE inverse hexagonal phase
}

Kent, Benjamin; Garvey, Chris; Lenne, Thomas; Porcar, Lionel; Garamus, Vasil; Bryant, Gary

https://researchrepository.rmit.edu.au/esploro/outputs/9921857673901341/filesAndLinks?institution=61 RMIT_INST\&index=null

Kent, B., Garvey, C., Lenne, T., Porcar, L., Garamus, V., \& Bryant, G. (2010). Measurement of glucose exclusion from the fully hydrated DOPE inverse hexagonal phase. Soft Matter, 6(6), 1197-1202. https://doi.org/10.1039/b919086d

Document Version: Accepted Manuscript

Published Version: https://doi.org/10.1039/b919086d

Repository homepage: https://researchrepository.rmit.edu.au

(C) Royal Society of Chemistry 2010

Downloaded On 2023/04/26 23:00:34 +1000

Please do not remove this page 
Thank you for downloading this document from the RMIT Research Repository.

The RMIT Research Repository is an open access database showcasing the research outputs of RMIT University researchers.

RMIT Research Repository: http://researchbank.rmit.edu.au/

\section{Citation:}

Kent, B, Garvey, C, Lenne, T, Porcar, L, Garamus, V and Bryant, G 2010, 'Measurement of glucose exclusion from the fully hydrated DOPE inverse hexagonal phase', Soft Matter, vol. 6 , no. 6 , pp. 1197-1202.

See this record in the RMIT Research Repository at:

http://researchbank.rmit.edu.au/view/rmit:5612

Version: Accepted Manuscript

Copyright Statement: (c) Royal Society of Chemistry 2010

Link to Published Version:

http://dx.doi.org/10.1039/b919086d 


\title{
Measurement of glucose exclusion from the fully hydrated DOPE inverse hexagonal phase
}

\author{
Ben Kent, ${ }^{a}$ Christopher J. Garvey, ${ }^{b}$ Thomas Lenné, ${ }^{c}$ Lionel Porcar, ${ }^{d}$ Vasil M. Garamus, ${ }^{e}$ and Gary \\ Bryant*a
}

The degree of exclusion of glucose from the inverse hexagonal $\mathrm{H}_{\mathrm{II}}$ phase of fully hydrated DOPE is determined using contrast variation small angle neutron scattering and small angle X-ray scattering. The presence of glucose is found to favour the formation of the non-lamellar $\mathrm{H}_{\mathrm{II}}$ phase over the fluid lamellar phase over a wider range of temperatures, while having no significant effect on the structure of the $\mathrm{H}_{\text {II }}$ phase. Glucose is preferentially excluded from the lipid/water interface resulting in a 10 glucose concentration in the $\mathrm{H}_{\text {II }}$ phase of less than half that in the coexisting aqueous phase. The degree of exclusion is quantified and the results are consistent with a hydration layer of pure water adjacent to the lipid headgroups from which glucose is excluded. The osmotic gradient created by the difference in glucose concentration is determined and the influence of glucose on the phase behaviour of non-lamellar phase forming lipid systems is discussed.

\section{Introduction}

15 Dehydration of biological tissue can induce phase transitions in cellular lipid membranes that affect the ability of the membrane to act as a semi-permeable barrier and are a pathway to irreversible damage to the cell ${ }^{1}$. These phase transitions can result in changes to the fluidity of the lipid hydrobcarbon tails (eg the fluid-gel transition), or cause the rearrangement of the lipids from a fluid lamellar $\left(\mathrm{L}_{a}\right)$ bilayer structure into non-bilayer structures such as the inverse hexagonal $\left(\mathrm{H}_{\mathrm{II}}\right)$ phase. The $\mathrm{H}_{\text {II }}$ phase (figure 1), which consists of lipid headgroups surrounding cylindrical water cores, arranged on a two-dimensional hexagonal lattice, is ${ }_{20}$ potentially lethal to cells as it cannot provide the semi-permeable barrier critical to cell function. This phase exists in a wide range of model lipid systems ${ }^{2,3}$ and is particularly prevalent in systems of dioleoylphosphatidylethanolamine (DOPE) $)^{4-6}$.

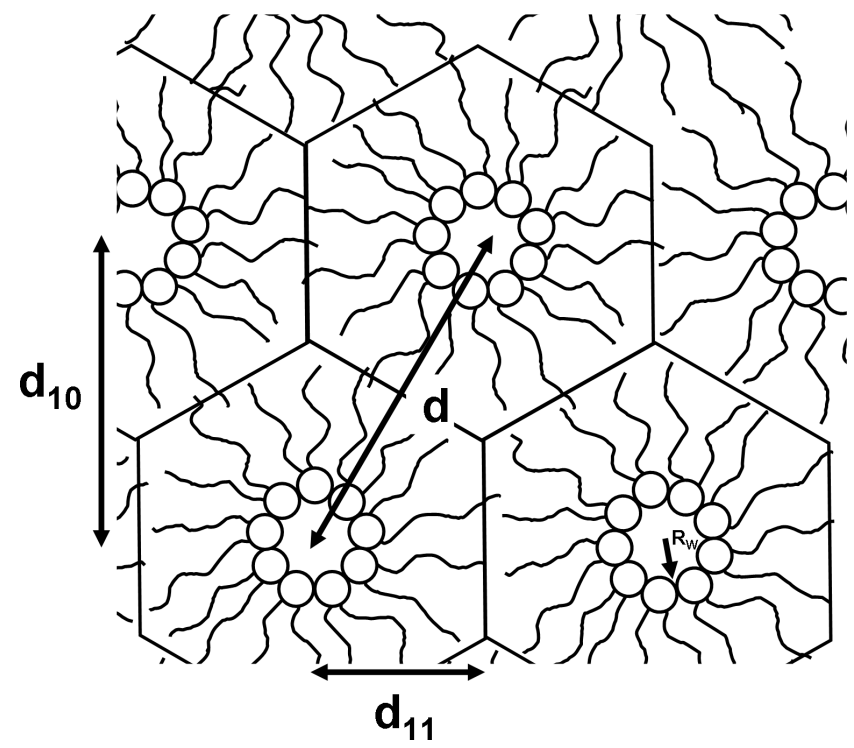

Fig. 1 Schematic diagram of the $\mathrm{H}_{\text {II }}$ phase.

Some organisms and organelles have developed defences against dehydration damage, one of which is the accumulation of ${ }_{25}$ sugars which have been shown to have cryoprotective properties in these systems ${ }^{7,8}$. The effects of sugars on the fluid-gel transition have been widely studied, and the mechnisms of protection are now reasonably well understood ${ }^{9}$. However, the effects of sugars on transitions to non-bilayer phases have been less well studied. Interestingly, and somewhat counter to their cryprotective nature, sugars have been shown to increase the propensity of a fully hydrated DOPE system to form the $\mathrm{H}_{\mathrm{II}}$ phase at the expense of the $\mathrm{L}_{\alpha}$ phase, in some cases completely suppressing the formation of the $\mathrm{L}_{\alpha}$ phase ${ }^{10,11}$. Above a limiting hydration 30 (known as full hydration), excess water is excluded from lipid mesophases into coexisting aqueous phases ${ }^{6}$. It has been established in lamellar systems that sugars are unequally partitioned between the membrane and excluded phases ${ }^{12-14}$. Therefore, to fully understand the mechanisms by which sugars affects the phase behaviour of $\mathrm{H}_{\mathrm{II}}$ forming lipid systems, it is necessary to determing the partitioning of the sugar between the $\mathrm{H}_{\mathrm{II}}$ cores and the excluded phase.

The complexity of natural biological membranes, which comprise many lipid species (as well as proteins) makes the 35 elucidation of specific effects difficult. Here we choose to use a model system, DOPE, as its PE headgroup and unsaturated acyl chains are prevalent among lipids in natural membranes. This lipid also readily self assembles into the inverse hexagonal phase 
over a wide temperature range. To study the effects of the sugars we choose a ratio of 0.5 glucose molecules per lipid, which is sufficiently high to affect the phase behaviour, but not high enough to introduce the complication of glass formation [ref].

In this paper, we employ small angle X-ray scattering to assign a lipid phase and combine this observation with an analysis of contrast variation small angle neutron scattering ${ }^{12}$ to quantitively determine the partitioning of glucose in a DOPE/water/glucose 5 system between a fully hydrated $\mathrm{H}_{\mathrm{II}}$ lipid phase and the coexisting excluded phase.

\section{Materials and Methods}

DOPE was obtained from Avanti Polar Lipids and Sigma Aldrich and used without further purification. Deuterated D-glucose- $\mathrm{d}_{7}$ was obtained from Sigma Aldrich. Two sample compositions were studied - DOPE fully hydrated in water and DOPE fully

10 hydrated in a water/glucose solution with a lipid:glucose molar ratio 1:0.5. The lipid volume fraction of each sample was 0.5 . Dry DOPE was measured by weight with water and glucose solution added volumetrically to each sample to achieve the desired lipid volume fraction and glucose ratio. Samples were mixed by a combination of vortex mixing with repeated temperature cycling through the $\mathrm{L}_{\beta} / \mathrm{L}_{\alpha}-\mathrm{H}_{\text {II }}$ phase transtions. Sample mixing was assessed both visually, and by the observation of the reproducibility of measurements (see below).

15 Small angle X-ray scattering (SAXS) experiments were performed on a Bruker Nanostar covering the q range 0.035 to 0.85 $\AA^{-1}$. Samples were transferred to aluminium samples holders with windows of $3 \mathrm{M}$ clear adhesive tape, which provides very low intensity uniform background scattering. Temperature control was achieved using a circulating water chiller/heater. Samples were equilibrated for at least 15 minutes prior to each measurement. Exposure times were 2 hours. Longer measurements were made at $25{ }^{\circ} \mathrm{C}$ on samples in $2 \mathrm{~mm}$ quartz capillaries (Wolfgang Muller Glas Technik, Berlin) sealed using Araldite expoxy resin. Samples 20 were returned to their initial measurement temperature and re-measured following each measurement program to check for lipid degradation or radiation damage. No evidence of damage was found, and all measurements were found to be consistent. Additionally, measurements using the alumnium sample holder were consistent with those using the capillaries.

Preliminary SANS measurements were performed on the SANS-1 instrument at the Geesthacht Neutron Facility (Geesthacht, Germany). Final SANS measurements were conducted on the NIST Center for Neutron Research NG7 SANS instrument 25 (Gaithersburg, Maryland). Data was collected on a 2D detector at sample to detector distances of $1 \mathrm{~m}, 4.5 \mathrm{~m}$ and $13.5 \mathrm{~m}$, giving a combined q range of $3.685 \times 10^{-3}$ to $0.03 \AA^{-1}$. Each sample composition was prepared at five $\mathrm{D}_{2} \mathrm{O} / \mathrm{H}_{2} \mathrm{O}$ ratios: $0,20,40,60$ and $80 \% \mathrm{D}_{2} \mathrm{O}$. Samples were mounted in quartz demountable cells (Hellma, Germany, 106QS, path length $0.2 \mathrm{~mm}$ ). These cells were mounted inside titanium demountable cell holders with quartz windows and sealed with viton o-rings. The temperature of the samples was kept constant at $25^{\circ} \mathrm{C}$ using a Julabo (Julabo Labortechnik Seelbach, Germany) circulating bath filled with silicon

30 oil. Data was normalised to sample transmission, corrected for background, empty cell scattering and detector efficiency. A flat incoherent scattering component determined from the scattering intensity at high q was subtracted from the data.

\section{Results and discusion}

\section{SAXS results}

Figure 2 shows the scattering of the DOPE and DOPE/glucose samples in the temperature range -5 to $25{ }^{\circ} \mathrm{C}$. The phase of the 35 lipid was identified from the characteristic spacing of the higher order Bragg reflections. The lamellar $\mathrm{L}_{\alpha}$ phase peaks index to $\mathrm{q}_{\mathrm{n}}$ $=\mathrm{nq}_{1}$ while the $\mathrm{H}_{\mathrm{II}}$ phase indexes according to:

$$
\mathrm{q}_{\mathrm{hk}}=\sqrt{\left(\mathrm{h}^{2}+\mathrm{hk}+\mathrm{k}^{2}\right)}
$$



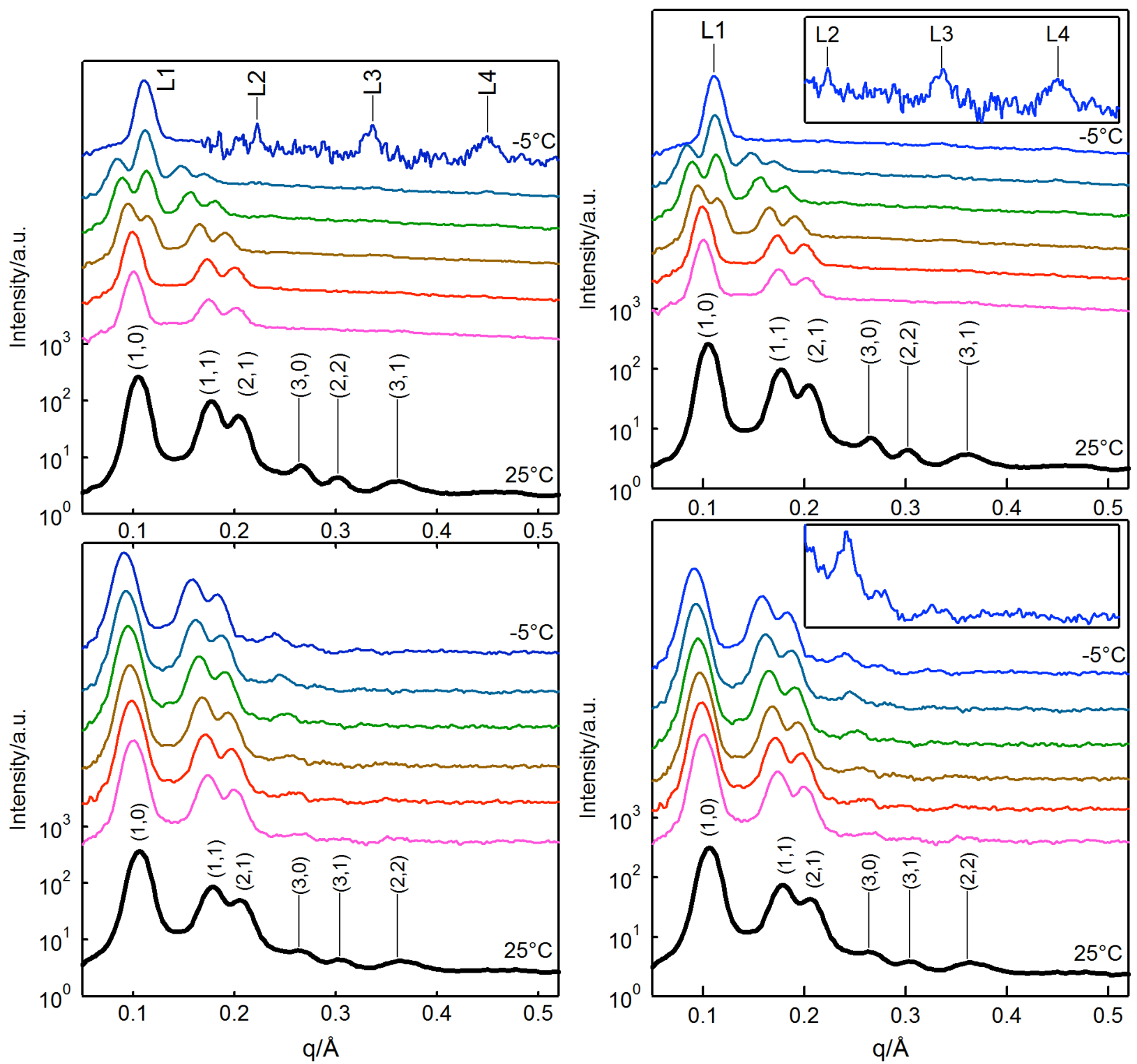

Fig. 2. SAXS measurements for the lipid/water system (top) and the lipid/water/glucose system (bottom). Temperature increases from top to bottom in 5 ${ }^{\circ} \mathrm{C}$ steps. Bragg reflection indices of $\mathrm{H}_{\text {II }}$ phase are shown in brackets. The insets show the $-5{ }^{\circ} \mathrm{C}$ data on an expanded linear vertical scale.

Up to six peaks were visible in the longer SAXS measurements, with generally only the first three peaks visible in the short 5 runs. The $\mathrm{H}_{\mathrm{II}}-\mathrm{L}_{\alpha}$ phase transition was observed in the DOPE/water sample on cooling, with a coexistence region of the two phases between -5 and $10{ }^{\circ} \mathrm{C}$. With the addition of glucose, this transition was completely suppressed, with $\mathrm{H}_{\text {II }}$ observed over the accessible temperature range. The lamellar phase is identified as the fluid lamellar $\mathrm{L}_{\alpha}$ phase. While the q-range does not extend to the wide angle region where examination of the intra-lipid peak would enable the direct determination of the degree of lipid chain fluidity, the transition temperature and $d$ spacing of the phase $(55 \AA)$ both indicate the $\mathrm{L}_{\alpha}$ phase. This was confirmed by 10 differential scanning calorimetry (not shown) which showed a transition peak with the characteristics of a $\mathrm{H}_{\text {II }}-\mathrm{L}_{\alpha}$ phase transition.

Electron density profiles were reconstructed for each system at $25^{\circ} \mathrm{C}$ using Fourier analysis of the SAXS data ${ }^{15}$. The amplitude of each Bragg peak was found by calculating the area of a fitted Gaussian curve to the background subtracted SAXS peak. Each amplitude was corrected for the multiplicity factor of the peak. A Lorentz correction was applied by dividing the peak intensity by

15 the magnitude of the reciprocal lattice vector. Finally the amplitudes were normalised to the amplitude of the $(1,0)$ peak. The phase of each amplitude, reduced to either positive or negative due to the centro-symmetry of the $\mathrm{H}_{\mathrm{II}}$ cell, was $+_{-}+{ }_{+}^{+}+$as determined by Harper et. al. ${ }^{15}$. This has consistently been the phasing of choice for the $\mathrm{H}_{\mathrm{II}}$ lipid phase, and gave the most physical result for both systems studied here. The electron density profiles determined for each system using this phasing are shown in Figure 3. They are essentially identical, the presence of glucose slightly reducing the d spacing from $74.0 \AA$ to $73.8 \AA$ 20 (note that regardless of which phasing was chosen, the two profiles were identical). 


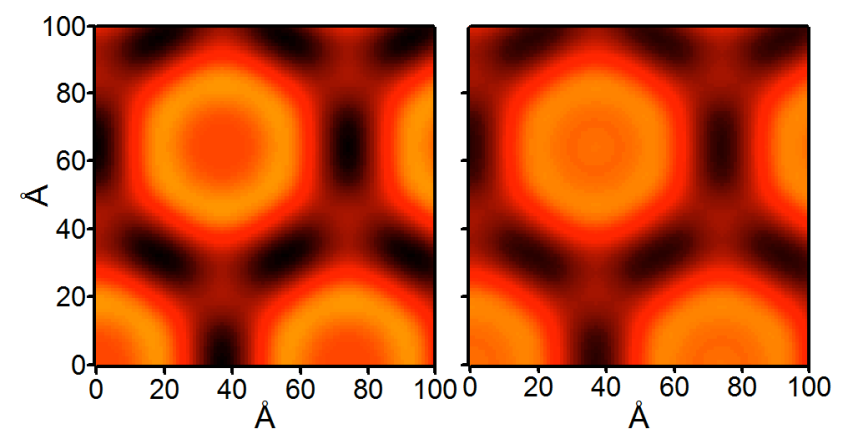

Fig. 3. Electron density profiles of the two systems studied. Lipid/water (left) and lipid/glucose/water (right)

The position of maximum electron density relative to the centre of the water core $\left(\rho_{\max }\right)$, (otherwise known as the Luzzati ${ }_{5}$ boundary $\left.{ }^{16}\right)$, was found to be the same for both systems within the errors: $19 \pm 0.5 \AA$. The radius of the water core $\left(\mathrm{R}_{\mathrm{w}}\right) \mathrm{can}$ be determined from this value with varying degrees of accuracy depending on the number of SAXS peaks observed and their resolution. Limited resolution systematically causes $\rho_{\max }$ to be lower than $\mathrm{R}_{\mathrm{w}}{ }^{17,}{ }^{18}$. Gravimetric measurements by Tate et. al. ${ }^{6}$ of the DOPE/water system found the radius of the water core in fully hydrated DOPE $\mathrm{H}_{\text {II }}$ systems to be $21 \AA$ at $25^{\circ} \mathrm{C}^{6}$. Given the similarities in the electron densities of the systems in this study, it is reasonable to assume this value is also applicable to the $10 \mathrm{lipid/water/glucose} \mathrm{system.}$

The radius of the water core indicates that the volume fraction of the lipid in the $\mathrm{H}_{\mathrm{II}}$ phase is $\psi_{\mathrm{L}}=0.709$. Thus it is clear that not all of the solvent in each of the systems is being incorporated into the $\mathrm{H}_{\text {II }}$ phase and a coexisting phase of excluded solvent exists in equilibrium with the $\mathrm{H}_{\text {II }}$ phase, with the volume fraction of the $\mathrm{H}_{\text {II }}$ and excluded phases respectively 0.71 and 0.29 .

\section{${ }_{15}$ SANS results}

The method of using contrast variation SANS to determine the partioning of a solute between microphases in lipid systems was originally demonstrated by Deme and Zemb ${ }^{12}$. Briefly, two sample sets are studied - one with and one without the solute present. The contrast of the solvent (water) is varied by changing its $\mathrm{D}_{2} \mathrm{O} / \mathrm{H}_{2} \mathrm{O}$ ratio, and the point at which the contrast of the solvent is equal to the contrast of the rest of the sample is identified - this is called the contrast match point (CMP). Considering a fully 20 hydrated lipid $\mathrm{H}_{\text {II }}$ phase in a glucose/water solution, at the CMP the sum of the scattering from the membrane phase (lipid and the water and glucose in the $\mathrm{H}_{\text {II }}$ cores) matches the total scattering from the excess glucose/water excluded phase. Therefore at the CMP the sum of the scattering length densities (SLDs) of each of the components in the two microphases, weighted by their volume fractions, must be equal:

$$
\rho_{\mathrm{L}} \psi_{\mathrm{L}}+\rho_{\mathrm{S}} \psi_{\mathrm{S}}+\rho_{\mathrm{W}} \psi_{\mathrm{W}}=\rho_{\mathrm{S}} \psi_{\mathrm{S}}^{\prime}+\rho_{\mathrm{W}} \psi_{\mathrm{W}}^{\prime}
$$

${ }_{25}$ where: $\rho_{\mathrm{L}}, \rho_{\mathrm{S}}$, and $\rho_{\mathrm{W}}$ are the scattering length densities of the lipid, solute (glucose) and water respectively; $\psi_{\mathrm{L}}, \psi_{\mathrm{S}}, \psi_{\mathrm{W}}$, are the volume fractions in the lipid phase; and $\psi_{\mathrm{S}}^{\prime}, \psi_{\mathrm{w}}^{\prime}$ are the solute and water volume fractions in the excluded phase; and the sum of the volume fractions in each phase must be unity:

$$
\begin{array}{r}
\psi_{\mathrm{L}}+\psi_{\mathrm{S}}+\psi_{\mathrm{W}}=1 \\
\psi_{\mathrm{S}}^{\prime}+\psi_{\mathrm{W}}^{\prime}=1
\end{array}
$$

For a three component system consisting of lipid, glucose and water, the global volume fraction of each component is related to 30 the local volume fractions in each phase by the following relationships:

$$
\begin{aligned}
& \Phi_{\mathrm{L}}=\psi_{\mathrm{L}} v \\
& \Phi_{\mathrm{W}}=\psi_{\mathrm{W}} v+\psi_{\mathrm{W}}^{\prime}(1-v) \\
& \Phi_{\mathrm{S}}=\psi_{\mathrm{S}} v+\psi_{\mathrm{S}}^{\prime}(1-v) \\
& \Phi_{\mathrm{L}}+\Phi_{\mathrm{S}}+\Phi_{\mathrm{W}}=1
\end{aligned}
$$

where $v$ is the volume fraction of the $\mathrm{H}_{\text {II }}$ lipid phase relative to the total sample. 
The SANS scattering results for the lipid/water and lipid/water/glucose systems are shown in figure 4 on double logarithmic plots. Similar SANS curves are observed for both systems, with a change in the contrast match point (ie the minimum scattering occurs at a different $\mathrm{D}_{2} \mathrm{O} / \mathrm{H}_{2} \mathrm{O}$ ratio). The peak at high q corresponds to the main Bragg reflection observed in the SAXS experiments. The aim of contrast variation is to obtain the average scattering length density of the lipid phase. In classical contrast 5 variation this is done by plotting $(\mathrm{I}(0))^{1 / 2}$ vs contrast and determining the "match point" at which $(\mathrm{I}(0))^{1 / 2}=0[\mathrm{H}$. B. Stuhrmann, R. G. Kirste Z. Phys. Chem. 1967, 56, 334-341.]. Due to the large size of the aggregates in the hexagonal phase we can not measure I(0) directly. However, a similar analysis can be conducted by plottiong (I(q) $)^{1 / 2}$ vs contrast, which is valid as long as the match point is independent of q [ref Deme].

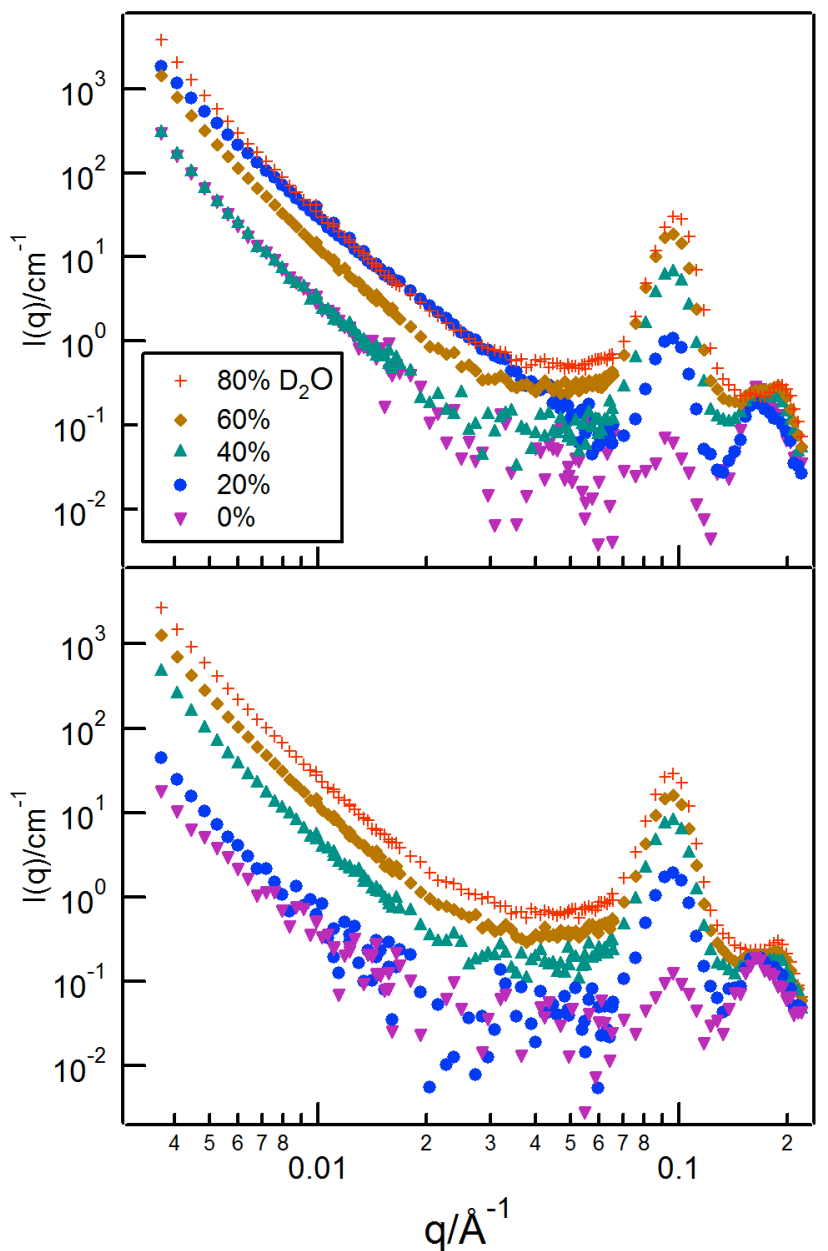

10 Fig. 4 SANS curves of lipid/water (top) and lipid/water/glucose (bottom) for different $\mathrm{D}_{2} \mathrm{O} / \mathrm{H}_{2} \mathrm{O}$ ratios. The measurement of $20 \%$ $\mathrm{D}_{2} \mathrm{O}$ lipid/water sample was inconsistent with other measurements and was disregarded in the analysis.

To extract the contrast match point, the square root of the intensity is plotted against the $\mathrm{D}_{2} \mathrm{O} / \mathrm{H}_{2} \mathrm{O}$ ratio for several values of $q$ in the small angle region $\left(\mathrm{q}<0.01 \AA^{-1}\right)$, as shown in figure 5. In both cases the contrast match points are independent of $\mathrm{q}$, negating the need to extrapolate to $\mathrm{I}(0)$. This yields vlaues for the CMP of $\Phi_{\mathrm{D} 20}=0.198$ and $\Phi_{\mathrm{D} 20}=0.092$ for the lipid/water and $15 \mathrm{lipid} /$ water/glucose samples respectively. Using the known SLDs for $\mathrm{D}_{2} \mathrm{O}$ and $\mathrm{H}_{2} \mathrm{O}$, this gives SLDs of $8.16 \times 10^{-7} \AA^{-2}$ and $7.94 \times 10^{-}$ ${ }^{8} \AA^{-2}$ respectively. These, along with the other quantities used in the analysis, are summarized in table 1.

For glucose-d7 equilibrated in a solution with $\Phi_{\mathrm{D} 20}=0.092$ the SLD is $5.74 \times 10^{-6} \AA^{-2}\left({ }^{19}\right)$. Solving for the local sugar volume fractions reveals $\psi_{\mathrm{S}}=0.0128$ and $\psi_{\mathrm{S}}=0.105$. From these local volume fractions the concentration of glucose in the excess solvent phase is found to be $c^{\prime}=0.105$ (by volume), where:

$$
\mathrm{c}^{\prime}=\frac{\psi_{\mathrm{s}}^{\prime}}{\psi_{\mathrm{s}}^{\prime}+\psi_{\mathrm{W}}^{\prime}}
$$

This is more than twice the concentration $\mathrm{c}=0.044$ in the aqueous channels in the $\mathrm{H}_{\text {II }}$ phase and is in line with previous studies which show partial exclusion of sugars from lipid lamellar phases ${ }^{12-14,20-22}$. The partition coefficient of $\mathrm{c} / \mathrm{c}^{\prime}=0.42$ is similar to the value of 0.5 found for DMPC bilayers ${ }^{14}$. 


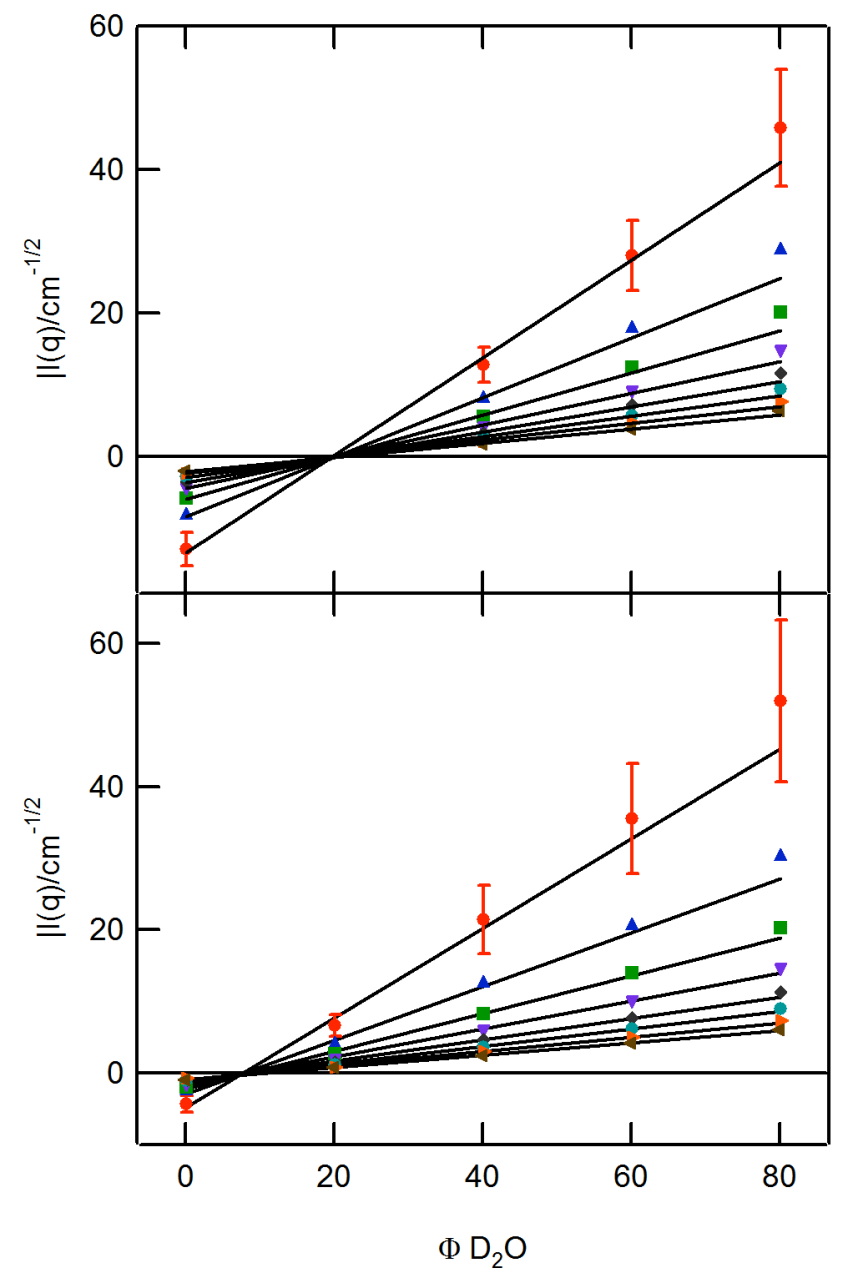

Fig. 5. Square root of intensity vs volume fraction of $D_{2} \mathrm{O}$ for several values of $\mathrm{q}$ in the small angle region. Shown are every second curve for $q$ values from $\mathrm{q}=3.685 \times 10^{-3} \AA^{-1}$ to $\mathrm{q}=0.01 \AA^{-1}$. The $\mathrm{q}$ independent contrast match points can be clearly seen for the lipid/water system (top) and the lipid/water/glucose system (bottom). Error bars are shown for $\mathrm{q}=3.685 \times 10^{-3} \AA^{-1}$.

${ }_{5}$ Clearly these results show that the glucose is partially excluded from the water channels in the $\mathrm{H}_{\text {II }}$ phase. The concentration remaining in the lipid phase corresponds to 0.12 glucose molecules per lipid (or 8.5 lipids per glucose molecule).

The bulk of evidence now suggests that the sugars are excluded from the region near lipid/water interface ${ }^{12,14,22}$ (the hydration layer). In the case of the $\mathrm{H}_{\text {II }}$ phase, this would imply that the glucose molecules are in the centre of the core (on average). Thus it is unlikely that the change in the phase behaviour of the DOPE/water system due to the presence of the glucose can be attributed 10 to direct interaction between the glucose molecules and the lipid polar headgroups. Assuming the concentration of sugar at the centre of the water core is the same as the concentration of the sugar in the aqueous phase, and knowing the $\mathrm{H}_{\text {II }}$ geometry, the depth of the hydration layer can be estimated. In this scenario, the overall sugar concentration in the $\mathrm{H}_{\mathrm{II}}$ phase (c=0.044) is split into a hydration layer of pure water adjacent to the lipid headgroups of depth $\sim 7.4 \AA$ and a cylinder of radius $\sim 13.6 \AA$ of sugar solution along the centre axis of the $\mathrm{H}_{\mathrm{II}}$ water channel with a sugar concentration $\mathrm{c}=0.105$. While in reality, no such discontinuity

15 in concentration exists, it is apparent from the size of the glucose molecules $r_{g}=3.5 \AA$ (approximating as a sphere) that this hydration layer depth is plausible. Knowing the area per lipid at the lipid/water interface $\left(48 \AA^{2}\right.$ reference $\left.{ }^{6}\right)$ there are about 9.8 water molecules in the hydration layer per lipid. 


\begin{tabular}{|c|c|c|c|}
\hline Quantity & $\begin{array}{c}\text { Lipid/Water/ } \\
\text { Glucose } \\
\text { system }\end{array}$ & $\begin{array}{c}\text { Lipid/Water } \\
\text { system }\end{array}$ & Comment \\
\hline$\Phi_{\mathrm{L}}$ & 0.5 & 0.5 & Sample prep \\
\hline$\Phi_{\mathrm{S}}$ & 0.04 & -- & Sample prep \\
\hline$\Phi_{\mathrm{W}}$ & 0.46 & 0.5 & Sample prep \\
\hline CMP & 0.092 & 0.198 & Figure 5 \\
\hline SLD at CMP & $7.94 \times 10^{-8} \AA^{-2}$ & $8.16 \times 10^{-7} \AA^{-2}$ & Figure 5 \\
\hline$\psi_{\mathrm{L}}$ & 0.709 & 0.709 & Reference $^{6}$ \\
\hline$\psi_{\mathrm{S}}$ & 0.0128 & -- & \\
\hline$\psi_{\mathrm{W}}$ & 0.278 & 0.291 & \\
\hline$\psi_{\mathrm{S}}^{\prime}$ & 0.105 & -- & \\
\hline$\psi_{W}^{\prime}$ & .895 & 1 & \\
\hline $\mathrm{v}$ & 0.71 & 0.71 & \\
\hline $\mathrm{n}_{\mathrm{w}} / \mathrm{n}_{1}$ & 15.9 & 16.65 & \\
\hline $\mathrm{n}_{\mathrm{s}} / \mathrm{n}_{1}$ & 0.12 & -- & \\
\hline $\mathrm{c}$ & 0.044 & -- & \\
\hline$c^{\prime}$ & 0.105 & -- & \\
\hline $\mathrm{V}_{\text {DOPE }}$ & $\begin{array}{r}1217 \\
30\end{array}$ & $\begin{array}{l}.3 \AA^{3} \\
\AA^{3}\end{array}$ & \\
\hline $\mathrm{V}_{\mathrm{H} 2 \mathrm{O}}$ & 186 & $5 \AA^{3}$ & Reference ${ }^{23}$ \\
\hline
\end{tabular}

5 The existence of the hydration layer therefore restricts the concentration of sugar in the $\mathrm{H}_{\text {II }}$ phase relative to the excess solvent. This difference creates an osmotic force which acts to remove water from the phase due to the difference in osmotic pressure of the solvents. Increasing the overall sugar concentration in the system would therefore be expected to create a larger osmotic difference between the $\mathrm{H}_{\text {II }}$ phase and the excess solvent and reduce the d spacing of the $\mathrm{H}_{\text {II }}$ phase. While the osmotic pressure difference in the systems studied here is too small to have an effect on the structural parameters of the $\mathrm{H}_{\text {II }}$ phase (1.05 MPa in the ${ }_{10} \mathrm{H}_{\mathrm{II}}$ phase and $2.74 \mathrm{MPa}$ in the excess solution ${ }^{23}$ ), Tenchov et. al. demonstrated this dehydrative behaviour in DHPE/water/sucrose systems $^{24}$.

\section{Conclusions}

The results show that the addition of glucose to a fully hydrated DOPE $\mathrm{H}_{\text {II }}$ phase has no effect on the structure of the phase. Glucose is incorporated into the phase albeit at a lower concentration than in the surrounding excess solvent. This exclusion is 15 consistent with the existence of a hydration layer of pure water adjacent to the lipid headgroups in the $\mathrm{H}_{\text {II }}$ phase from which the glucose molecules are excluded, thus indicating a preference for the lipid headgroups to associate with water over glucose molecules.

A consequence of the glucose concentration imbalance is the existence of an osmotic gradient between the $\mathrm{H}_{\text {II }}$ phase and the excess solvent which acts to remove water from the phase and favour the non-lamellar $\mathrm{H}_{\mathrm{II}}$ phase over the lamellar $\mathrm{L}_{\alpha}$ phase, due to 20 its smaller area per lipid at the lipid/water boundary. This is evident in the thermotropic behaviour of the DOPE/water/glucose system which favours the $\mathrm{H}_{\text {II }}$ phase over the fluid lamellar $\mathrm{L}_{\alpha}$ phase over a wider range of temperatures than an equivalent system without glucose.

These results highlight the complexity of the elucidation of the mechanisms with which sugars act as protectants against dehydration in natural organisms. Dehydration induced transitions such as the $\mathrm{L}_{\alpha}-\mathrm{H}_{\mathrm{II}}$ bilayer to non-bilayer transition, can ${ }_{25}$ destroy the semi-permeability of the cell membrane and are thus potentially lethal to cells. So the preference of the glucose system for the formation of the $\mathrm{H}_{\mathrm{II}}$ phase over the bilayer $\mathrm{L}_{\alpha}$ phase sits in contrast with the observed ability of sugars to prevent damage to biological cells during dehydration.

While it is true that lipids with the PE headgroup are directed towards the inner monolayer in healthy mammalian cells and thus away from the extra-cellular solution [ref], the changes in the forces acting on membranes during dehydration can lead to lipid 30 demixing [ref Bryant]. Therefore it is plausible that sugars and membrane regions rich in PE lipids are brought into close proximity, and it is instructive to know how they interact.

The preference for the $\mathrm{H}_{\text {II }}$ phase occurs in spite of the fact that most of the sugar is excluded from the $\mathrm{H}_{\text {II }}$ phase in a manner which suggests no direct involvement between the sugar and the lipids. Instead, with the hydration layer of pure water separating 
the lipid headgroups from the sugar molecules, non-specific properties of the sugar such as volumetric and osmotic effects during dehydration seem to be of greater significance.

\section{Notes and references}

${ }^{a}$ Applied Physics, RMIT University, Melbourne, Australia.

$5^{b}$ National Deuteration Facility, Australian Nuclear Science and Technology Organisation, Menai, Australia

${ }^{c}$ Functional Ecology Group, Research School of Biology, The Australian National University, Canberra, Australia.

${ }^{d}$ Institut Laue-Langevin, Grenoble, France

${ }^{d}$ GKSS Research Centre, Geesthacht, Germany.

e Applied Physics, RMIT University, Melbourne, Australia. Fax: +61 39925 5290; Tel: +61 39925 2139; E-mail: gary.bryant@rmit.edu.au 10

1. J. Wolfe and G. Bryant, in NATO ASI Series Vol H 64 Mechanics of Swelling, ed. T. K. Karalis, Springer-Verlag, Berlin Heidelberg, 1992.

2. J. M. Seddon, Biochimica et Biophysica Acta (BBA) - Reviews on Biomembranes, 1990, 1031, 1-69.

3. M. S. Webb, H. Sek Wen and P. L. Steponkus, Biochimica et Biophysica Acta (BBA) - Biomembranes, 1993, 1145, 93-104.

4. S. M. Gruner, M. W. Tate, G. L. Kirk, P. T. C. So, D. C. Turner and D. T. Keane, Biochemistry, 1988, 27, 2853-2866.

15 5. E. Y. Shalaev and P. L. Steponkus, Biochimica et Biophysica Acta (BBA) - Biomembranes, 1999, 1419, 229-247.

6. M. W. Tate and S. M. Gruner, Biochemistry, 1989, 28, 4245-4253.

7. J. H. Crowe, L. M. Crowe and D. Chapman, Science, 1984, 223, 701-703.

8. S. Calderon, M. Holmstrup, P. Westh and J. Overgaard, Journal of Experimental Biology, 2009, 212, 859-866.

9. T. Lenne, G. Bryant, R. Holcomb and K. L. Koster, Biochimica et Biophysica Acta (BBA) - Biomembranes, 2007, 1768, 1019-1022.

20 10. R. D. Koynova, B. G. Tenchov and P. J. Quinn, Biochimica Et Biophysica Acta, 1989, 980, 377-380.

11. C. A. Wistrom, R. P. Rand, L. M. Growe, B. J. Spargo and J. H. Crowe, Biochimica et Biophysica Acta (BBA) - Biomembranes, $1989,984,238-242$.

12. B. Demé and T. Zemb, Journal of Applied Crystallography, 2000, 33, 569-573.

13. T. Lenne, G. Bryant, C. J. Garvey, U. Keiderling and K. L. Koster, Physica B: Condensed Matter, 2006, 385-386, 862-864.

14. P. Westh, Physical Chemistry Chemical Physics, 2008, 10, 4110-4112.

25 15. P. E. Harper, D. A. Mannock, R. N. A. H. Lewis, R. N. McElhaney and S. M. Gruner, Biophysical Journal, 2001, 81, $2693-2706$.

16. V. Luzzati, in Biological Membranes: Physical Fact and Function, ed. D. Chapman, Academic Press, London, 1968, pp. 71-123.

17. M. Rappolt, A. Hickel, F. Bringezu and K. Lohner, Biophysical Journal, 2003, 84, 3111-3122.

18. D. C. Turner and S. M. Gruner, Biochemistry, 1992, 31, 1340-1355.

19. V. F. Sears, Neutron News, 1992, 3, 26 - 37.

30 20. G. Bryant and K. L. Koster, Colloids and Surfaces B: Biointerfaces, 2004, 35, 73-79.

21. K. L. Koster, K. J. Maddocks and G. Bryant, European Biophysics Journal, 2003, 32, 96-105.

22. F. Pincet, E. Perez and J. Wolfe, Cryobiology, 1994, 31, 531-539.

23. K. Kiyosawa, Bull. Chem. Soc. Jpn., 1988, 61, 633-642.

24. B. Tenchov, M. Rappolt, R. Koynova and G. Rapp, Biochimica et Biophysica Acta (BBA) - Biomembranes, 1996, 1285, 109-122. 